\title{
The influence of the droplet clouds microstructure on the polarization characteristics of a double scattering lidar signal
}

\author{
Yevgeniy Nee, Valentina Bryukhanova, and Anton Doroshkevich \\ Tomsk State Universities, Radiophysics Department, Russian Federation
}

\begin{abstract}
The results of calculation of the ratio of polarization degrees of the double scattering lidar return from droplet clouds with different microstructure at sensing by circularly and linearly polarized radiation are given in this report. The influence of the droplet size on ellipse parameters of linearly polarized radiation are discussed.
\end{abstract}

\section{Introduction}

Determination of the clouds microstructure is significant scientific and practical interest. This is explained by the clouds make significant impact on the radiation balance of the Earth. The lidar allows us to study clouds without changes of them in near real-time with high spatial resolution $[1,2]$. Most often, the interpretation of the lidar data is based on the lidar equation, which was got for the assumption that sensing of the atmosphere is limited by the contribution of photons scattered on the receiving system direction only the once. This approach is justified for the aerosol formation with small optical depth such as lowdensity haze and high-level clouds. However, the multiple scattering makes significant contribution to the lidar return at the study of many types of low-level and medium-level clouds, fogs, dense hazes.

Experimental studies of brightness and polarization characteristics of the multiple backscattering radiation [3] showed that the intensity of the multiple scattering signal is distributed over the full field-of-view of the lidar receiving system. Single scattering lidar return is formed in the space determined by the radiation pattern of the lidar source and doesn't depend on the lidar field-of-view. It is showed in the paper [4], the value of multiple scattering lidar return associates with the generalized parameter $\eta=H \sigma \operatorname{tg}(\theta / 2)$, which is determined by scattering coefficient $(\sigma)$ and cross-section of the scattering volume $(H \operatorname{tg}(\theta / 2))$. So, the multiple scattering lidar return is formed in the scattering volume limited by the field-of-view of lidar receiving system: more the receiving system field-of-view, more the cross-section of scattering volume and, therefore, more the multiple scattering contribution in the lidar return. Thus, the changes of power of the lidar return by increasing the receiving system field-of-view of lidar occurs only due to the increase of multiple scattering signal. Therefore, the component of multiple scattering can be recovered from the lidar return by using lidar with multiple field-of-view [5-8]. 
The use of the stop blocked single scattering radiation in the lidar receiver for registration of the multiple scattering lidar return was offered in the paper [5]. In this case, the photosensitive element receives only the multiple scattering radiation. Diaphragms with different diameter which was set in the receiving system by turns are used for the decomposition of a lidar return into single and multiple scattering components in [6, 7]. Using a CCD-camera [8] set in the focal plane of a receiving lens, is equivalent to carrying out the experiment with several receiving channels with different field-of-view.

The multiple scattering coaxial lidar is developed for a detailed study of the lidar return structure from tropospheric clouds by the OES\&RS department of TSU. The CCD camera will be used as a receiver [9].

\section{Double scattering lidar return}

In many practically significant cases the lidar return can be described based on double scattering approximation with sufficient accuracy. Model of the double scattering lidar return was reported in the most details by Kaul and Samokhvalov [10].

The polarization state of lidar return can be conveniently represented in the form of the Stokes vector. The expression describing the Stokes vector of double scattering lidar return looks as follows [11]:

$$
\vec{S}(r)=\vec{S}^{(1)}(r)+\vec{S}^{(2)}(r)=\frac{P^{(1)}(r)}{X(\pi)}\left[\hat{M}(\pi) \vec{S}_{0}+2 \sigma_{0} r\left(\vec{I}_{1}+\vec{I}_{2}\right)\right],
$$

where

$$
\begin{gathered}
\vec{I}_{1}=\sigma_{0}(r-H) \int_{0}^{\gamma_{1}} \int_{0}^{2 \pi} \vec{G}(\varphi, \gamma, \pi-\gamma) \operatorname{tg} \frac{\gamma}{2} d \varphi d \gamma, \quad \vec{I}_{2}=\int_{\gamma_{1}}^{\pi / 2} \int_{0}^{2 \pi} \vec{G}(\varphi, \pi-\gamma, \gamma) d \varphi d \gamma, \\
\gamma_{1}=2 \operatorname{arctg} \frac{r \operatorname{tg} \frac{\theta_{0}}{2}}{r-H}, \\
\vec{G}(\varphi, \gamma, \pi-\gamma)=\hat{R}(\varphi) \hat{M}(\pi-\gamma) \hat{M}(\gamma) \hat{R}(\varphi) \vec{S}^{0}
\end{gathered}
$$

is the integral matrix vector-parameter defined by the operator of a references plane rotating on the angle $\varphi$ concerning base

$$
\hat{R}(\varphi)=\left(\begin{array}{cccc}
1 & 0 & 0 & 0 \\
0 & \cos 2 \varphi & \sin 2 \varphi & 0 \\
0 & -\sin 2 \varphi & \cos 2 \varphi & 0 \\
0 & 0 & 0 & 1
\end{array}\right)
$$

$\hat{M}$ - scattering phase matrix, which contains the maximum possible information on the scattering medium [12]. In the general case it comprises 16 components

$$
\hat{M}=\left(\begin{array}{llll}
f_{11} & f_{12} & f_{13} & f_{14} \\
f_{21} & f_{22} & f_{23} & f_{24} \\
f_{31} & f_{32} & f_{33} & f_{34} \\
f_{41} & f_{42} & f_{43} & f_{44}
\end{array}\right) .
$$

Elements of this matrix describe by the combination of equations for the amplitude of the scattered radiation field. Scattering phases matrix depends on the complex refractive index, particle size and shape [13]. 


\section{The dependence of the polarization degree of the double scattering lidar return from the water content of the cloud}

We use of droplet cloud models [14] been typical of the Kamchatka region [15] for calculation of polarization characteristics of the double scattering lidar return (tab.1). Optical characteristics of these clouds were calculated by using the program PolyMie [16].

Table 1. Parameters of the droplet clouds microstructure.

\begin{tabular}{|l|l|l|l|}
\hline $\boldsymbol{r}_{\boldsymbol{m}} \boldsymbol{\mu \mathbf { m }}$ & $\boldsymbol{\mu}$ & $\boldsymbol{N}, \mathbf{c M}^{-\mathbf{3}}$ & $\boldsymbol{\delta}, \mathbf{g} / \mathbf{m}^{\mathbf{3}}$ \\
\hline 4,48 & 10,6 & 350 & 0,220 \\
\hline 4,94 & 5,8 & 58 & 0,070 \\
\hline 4,94 & 7,2 & 96 & 0,100 \\
\hline 5,28 & 8,5 & 80 & 0,092 \\
\hline 5,46 & 2,8 & 59 & 0,194 \\
\hline 5,72 & 6,4 & 103 & 0,180 \\
\hline 5,95 & 3,4 & 39 & 0,133 \\
\hline 5,95 & 22,2 & 165 & 0,188 \\
\hline
\end{tabular}

Here, $r_{m}-$ modal radius of the particle size distribution, $\mu-$ half-width parameter, $N-$ particle number concentration, $\delta$-cloud liquid water content.

Analysis of calculation results shows that the ratio of polarization degrees of double scattering lidar return at sensing circularly and linearly polarized radiation doesn't depend on the lidar receiving system field-of-view (Fig.1). This allows us to speed up the processing of the experimental data by reducing amount of data read out from the CCDcamera and used for the interpretation of experimental data.

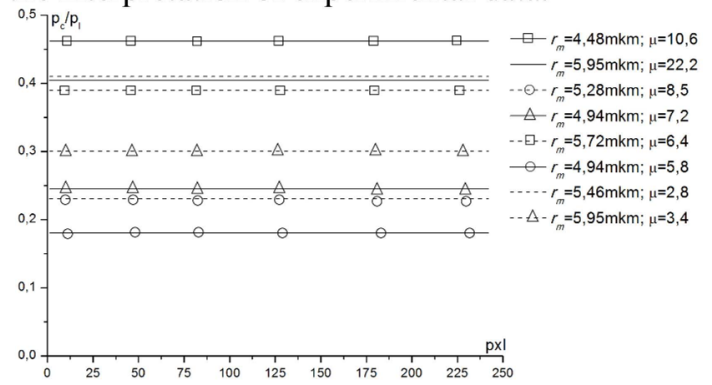

Fig. 1. The dependence of the ratio of polarization degrees of the double scattering lidar return at sensing circularly and linearly polarized radiation on the field-of-view of the lidar receiving system.

A more detailed research of the dependence of the ratio of polarization degrees of the double scattering lidar return on microstructure parameters of a cloud showed that the increase of the polarization degree ratio occurs with the increase of liquid water content of clouds (Fig. 2).

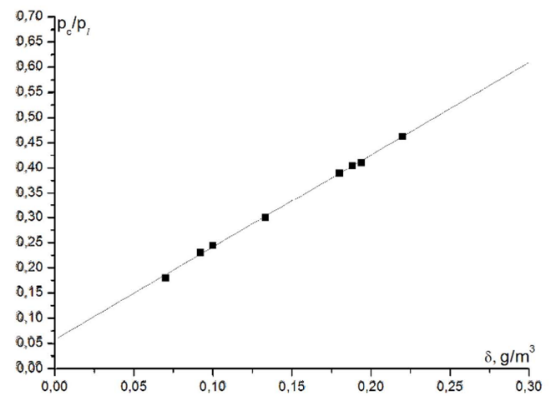

Fig. 2. The dependence of the ratio of polarization degrees of double scattering lidar return at sensing circularly and linearly polarized radiation on the water content of cloud been typical of the Kamchatka region. 
In the general case the dependence of the ratio of polarization degree at sensing circularly and linearly polarized radiation on the liquid water content of cloud is nonlinear [17] for all models of clouds reported in [14]. The slope angle of the approximating function for cloud models described above is $1.08 \pm 0.02$. As you can see cloud models been typical of Kamchatka region is in good agreement with the present trend.

\section{The influence of particle size on polarization ellipse parameters of the double scattering lidar return}

The study of the influence of sensing droplet cloudy microstructure on the polarization state of the double scattering lidar return calls to analyze the transformation of polarization state of sounding radiation.

Previously $[18,19]$, we calculated the distribution of the polarization degree and the elements of the normalized Stokes vector over the focal plane of the receiving system. These calculations were based on the expression (1). According to results the pattern of intensity distribution over the detection plane depends on the polarization state of sounding radiation. There are areas of prevailing depolarization and full polarization. If laser pulse is linearly polarized the areas of prevailing depolarization $\left(45^{\circ}, 135^{\circ}, 225^{\circ}\right.$ и $\left.315^{\circ}\right)$ and total polarization $\left(0^{\circ}, 90^{\circ}, 180^{\circ}\right.$ и $\left.270^{\circ}\right)$ will be observed. If laser pulse is circular polarized the distribution pattern is equal for all azimuthal angles [19].

In order to analyze the structure features of the polarization degree distribution we studied the distribution of the ellipticity $\varepsilon$ and the semi-major axis orientation of the ellipse of polarization $\varphi$ over the detection plane of the receiving system.

$$
\varepsilon=\frac{1}{2} \arcsin \left(\frac{S_{4}}{\sqrt{S_{2}^{2}+S_{3}^{2}+S_{4}^{2}}}\right), \quad \varphi=\frac{1}{2} \operatorname{arctg}\left(\frac{S_{3}}{S_{2}}\right) .
$$

According to (1) the Stokes vector of the double scattering lidar signal is determined by the size of the scattering volume, which depends on the distance to an aerosol formation of $\mathrm{H}$ and the receiving system field of view $\theta 0$, and by optical properties of this volume (scattering coefficient $\sigma$ and phase scattering matrix M).

We used continental droplet cloud models for a scattering media. To reduce the influence of geometrical and optical parameters of aerosol formation on the study results the selection criterion was the similar value of the cloud base $\mathrm{H}$ and the scattering coefficient $\sigma$. Chosen models lie in the line section in Fig. 2. The droplet number concentration $N$, mode radius $r_{\mathrm{m}}$, half-width parameter of the gamma particle size distribution $\mu$ and liquid water content $\delta$ of studied aerosol formation are shown in table 2 .

Table 2. The parameters of droplet cloud models.

\begin{tabular}{|c|c|c|c|c|c|c|}
\hline № & $\boldsymbol{H}, \mathbf{M}$ & $\boldsymbol{N}, \mathbf{c m}^{-\mathbf{3}}$ & $\boldsymbol{r}_{\mathbf{m}}, \mathbf{M K M}$ & $\boldsymbol{\mu}$ & $\boldsymbol{\delta}, \mathbf{\Gamma} / \mathbf{M}^{\mathbf{3}}$ & $\boldsymbol{\sigma}_{\mathbf{~}} \mathbf{K M}^{-\mathbf{1}}$ \\
\hline 1 & 840 & 680 & 2,68 & 13,0 & 0,08 & 19,59 \\
\hline 2 & 750 & 396 & 2,83 & 3,3 & 0,15 & 21,00 \\
\hline 3 & 908 & 480 & 3,49 & 14,8 & 0,12 & 20,01 \\
\hline 4 & 1000 & 250 & 4,38 & 6,2 & 0,20 & 20,19 \\
\hline 5 & 980 & 297 & 4,41 & 13,3 & 0,16 & 19,27 \\
\hline 6 & 950 & 165 & 5,95 & 22,2 & 0,19 & 20,69 \\
\hline
\end{tabular}

The particle size distributions of selected cloud models are presented in Fig. 3. Models 1 and 2 and also 4 and 5 have similar values of the modal radius but different halfwidth of the particle size distribution. On the other hand, the models 2 and 4 and also 5 and 6 have different modal radius but identical half-width of the distribution. Thus, these models allow us to evaluate the influence of the modal radius and the half-width of the 
particle size distribution on the distribution parameters of polarization characteristics of the double scattering lidar return in the detection plane.

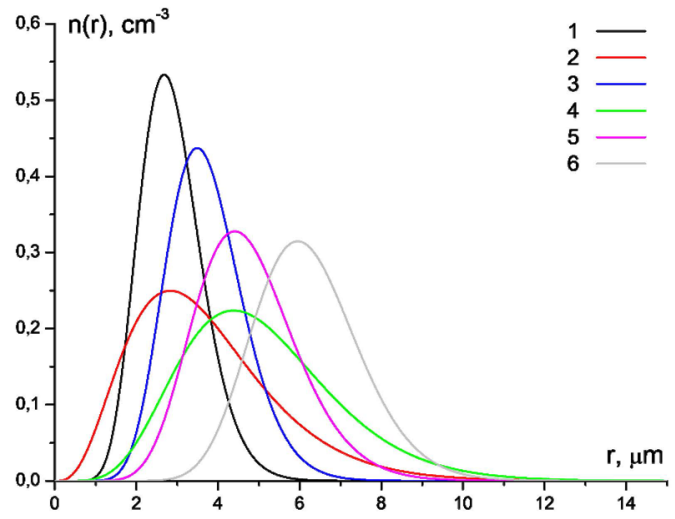

Fig. 3. The particle size distributions of droplet clouds.

The calculation was carried out for following parameters: the cloud base height is $1000 \mathrm{~m}$; the penetration depth of sounding pulse is $100 \mathrm{~m}$; wavelength of radiation is $532 \mathrm{~nm}$.

As shown by numerical simulation results (Fig. 4), the ellipticity $\varepsilon$ of the detected radiation of double scattering is sensitive to the microstructure parameters of the aerosol formation. The increasing of the half-width of the particle size distribution leads to the ellipticity $\varepsilon$ varies in the smaller range for the areas of prevailing depolarization. The azimuthal dependence of the ellipticity $\varepsilon$ are the same for models 2 and 4, and also 5 and 6 . It allows us to suggest the change of the modal radius of particles doesn't influence on the ellipticity $\varepsilon$.
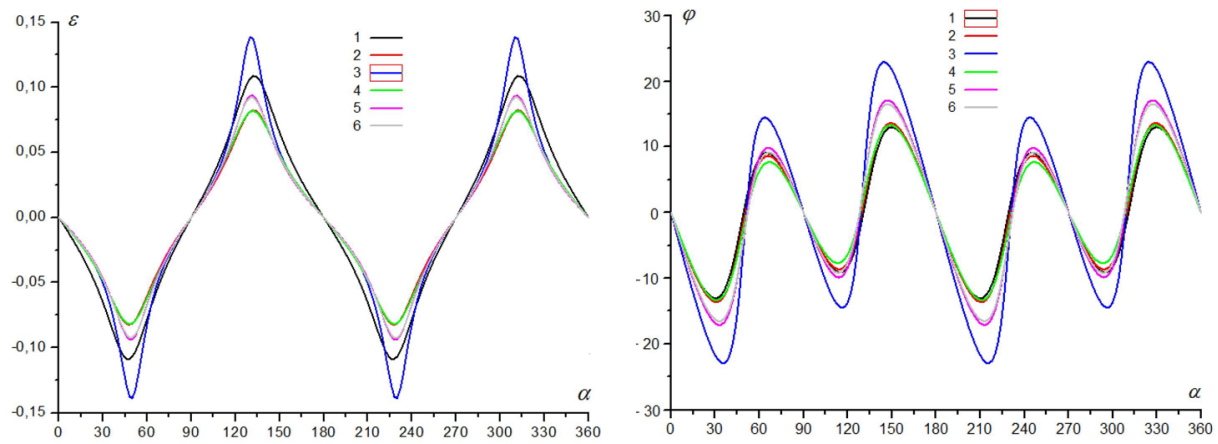

Fig. 4. Azimuthal distribution of a) the ellipticity $\varepsilon$ and b) the semi-major axis orientation $\varphi$ of the polarization ellipse of the double scattering lidar return over the receiver focal plane.

The azimuthal dependence of the semi-major axis orientation $\varphi$ of the polarization ellipse of the double scattering radiation is shown in Figure $4 \mathrm{~b}$. Obvious influence of the droplet cloud microstructure on the orientation of the polarization ellipse is not observed. At the same time clouds with similar values of the half-width of the particle size distribution demonstrate almost complete coincidence of the azimuthal dependence of the orientation of the polarization ellipse.

It's worth focusing on the droplet cloud model 3 (tab.2). The azimuthal dependence of considered polarization parameters of the double scattering radiation from this model shows the greatest deviation from zero. It indicates that this cloud model transforms the linear polarized sounding radiation to the elliptical polarized radiation more significant at the 
double scattering by cloud particles. The orientation of the semi-major axis of the polarization ellipse varies over a wider range than for other cloud models. This fact needs special research.

This research was supported by "The Tomsk State University Academic D.I. Mendeleev Fund Program” grant № 8.1.12.2015.

\section{References}

1. E.D. Hinkley, Laser monitoring of the atmosphere (New York, 1976)

2. M.R. Measures, Laser remote sensing (New York, 1984)

3. V.A. Donchenko, I.V. Samokhvalov, G.G. Matvienko, Atmospheric and oceanic physics, 7,11 (1971)

4. G.M. Krekov, S.I. Kavkianov, M.M. Krekova, Interpretation of the optical atmospheric sounding signals (Novosibirsk, 1987)

5. R. J. Allen and C. M. R. Plaft, Appl. Opt., 16, 12 (1977)

6. D.L. Hult, L.R. Bissonnette, L. Durand Appl. Opt., 33, 12 (1994)

7. V.V. Bryukhanova, I.V. Samokhvalov, A.I. Abramochkin, S.A. Abramochkin, A.A. Tikhomirov, Atmospheric and oceanic optics 16, 09 (2003)

8. L.I. Chaikovskaya, E.P. Zege, I.L. Katsev et al., Appl. Opt., 48, 623-632 (2009)

9. V.V. Bryukhanova, I.V. Samokhvalov, G.V. Simonova, Patent 1006336 Russian Federation, IPC G01S 17/88. The device registration lidar signal / the applicant and the patentee National Research Tomsk State University and the Institute of Monitoring of Climatic and Ecological Systems, Siberian Branch of the Russian Academy of Sciences. - published 20.12.10, bulletin № 35 . - 2 p.

10. B.V., Kaul, I.V., Samokhvalov, Optics atmosphere and ocean, 12, 18 (2005)

11. V.V. Bryukhanova, Proc. SPIE 9292, 20th International Symposium on Atmospheric and Ocean Optics: Atmospheric Physics, 92922C-3 (2014)

12. G.V.Rozenberg, Twilight (Moscow, 1963)

13. S.N. Volkov, I.V. Samokhvalov, D. Kim, JQSRT 123, 169-175 (2013).

14. A.A. Kokhanovsky, Cloud Optics (Springer, 2006)

15. Kamchatka Branch FGBI "AVIAMETTELEKOM ROSHYDROMET", Atlas of Clouds (2015)

16. Program PolyMie to calculate optical properties (phase function or matrix, extinction and absorption coefficients) of water droplet clouds, INTAS project 01-0239, May 2005, http://osmf.sscc.ru/ smp/INTAS 01-0239/main.html (17 may 2012).

17. Nee Y.V., V.V. Bryukhanova, A.A. Doroshkevich, News of Higher Schools. Physics., $58,8 / 2(2015)$

18. I.V. Samokhvalov, V.V. Bryukhanova, A.A. Doroshkevich and I.V. Zhivotenyuk, Rep. High School. Phisics, 55, 9/2 (2012)

19. A.A. Doroshkevich, V.V. Bryukhanova, I.V. Samokhvalov and A.P. Stykon, Proc. SPIE., 9292, 92922E (2014) 